\title{
Challenges to support Edge-as-a-Service
}

\author{
Steven Davy \\ Waterford Institute of Technology \\ Jeroen Famaey \\ Ghent University - iMinds
}

Joan Serrat and Juan Luis Gorricho
Universitat Politecnica de Catalunyna

\author{
Avi Miron \\ Technion \\ Israel Institute of Technology
}

\author{
Manos Dramitinos \\ Athens University of Economics and Business \\ Research Center
}

\author{
Pedro Miguel Neves \\ Portugal Telecom Inovacao SA
}

Steven Latré
University of Antwerp - iMinds

Ezer Goshen

BandWD 


\section{Telecommunications State of Play}

The access network is the most expensive part of an operators network and often comprises many different types of network equipment. This may include equipment to support cable modems, DSL broadband, 3G and 4G mobile broadband, Wifi and increasingly, optical network equipment to support Fibre-to-the-Home. Crucially, network operators that seek to offer mixed infrastructure, need to deploy multiple types of access network technologies, which is very costly and promotes inefficiency.

It is widely accepted that improving national and international Internet infrastructure will have a profoundly positive socio-economic impact ${ }^{1}$. The costs associated to these infrastructures are massive, and regulators are looking for ways to bring some stability to the situation. Specifically in Europe, recommendations on open access to next generation access networks ${ }^{2}$ is promoting the concepts of network sharing, and is encouraging incumbent network operators to open up their networks for new entrants. However, there is currently no leading technical solution to these recommendations.

A recent study ${ }^{3}$ highlights that there are several short term fixes that can help network operators control the cost of new network deployments, these include data offloading to $\mathrm{WiFi}$ from LTE, introducing more constrained data download capacities on users, more comprehensive traffic shaping through the use of Deep Packet Inspection (DPI), and introducing caching of content at the edge of networks. However, these short-term fixes are not sustainable into the future, and only innovation at the business level will allow network operators to maintain sustainable revenues. The EaaS concept not only supports the short term fixes, but introduces a new and innovative approach to reverse the trend of increasing CAPEX and OPEX. This is through the opening up and sharing of access network resources in a secure, intelligent and targeted way.

Current network sharing practices, such as those adopted by Mobile Virtual Network Operators (MVNO) are based on long term and rigid contractual agreements, which are both inflexible and time consuming to arrange. The MVNO model is beneficial for incumbent network operator as it promotes an increase in network revenue and a reduction in network costs.

We firmly believe that the EaaS concept can bring significant stability and innovation to the telecommunications market. We also see that the current MVNO models are too restrictive, based primarily on resale of network minutes and data. With EaaS, one can develop new ways for third parties to leverage the resources of large network operators and ultimately provide higher value to the end users.

\footnotetext{
${ }^{1}$ A Study on Socio-Economic Impact of Bandwidth http://ec.europa.eu/digital-agenda/en/news/study-socio-economic-impactbandwidth-smart-20100033, 2013

${ }^{2}$ Commission Recommendation of 20 September 2010 on regulated access to Next Generation Access Networks (NGA)

${ }^{3}$ Wireless Technology: Breaking the Limits of the TCO Reduction in Mobile Networks, Frost \& Sullivan, 2011
}

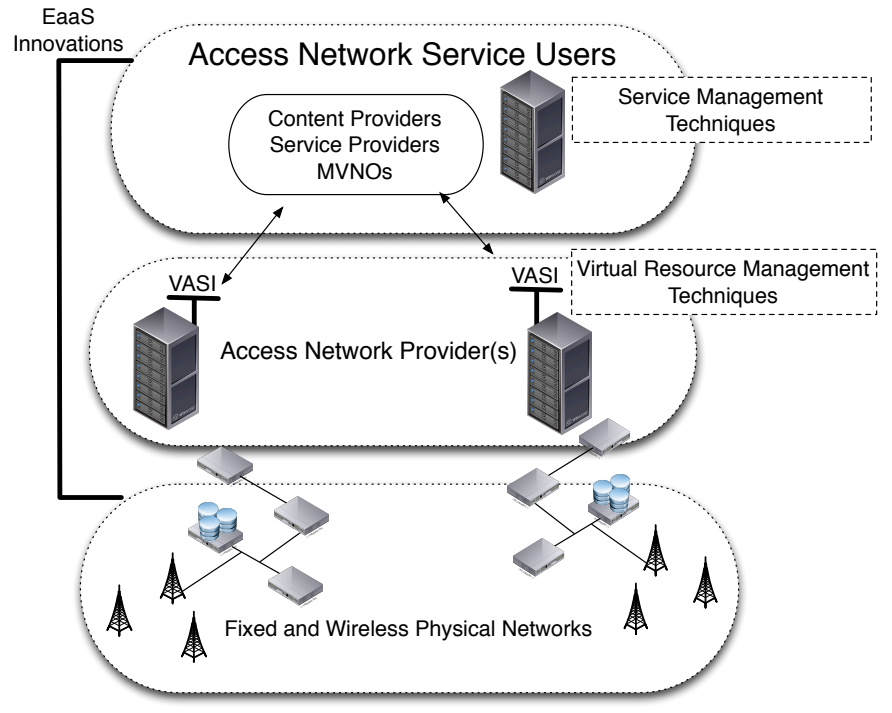

Figure 1. The Virtual Access Service Interface (VASI) can be used by content / service providers to request customisation of resources deep in network providers access networks. It is supported by advanced usage of network virtualisation and network programmability.

\section{A New NeTwork InTERFACE}

To realise the concept of EaaS, network operators need to be able to offer customised portions of their networks to services providers, and also a way to enable the further customisation of the network as new services are deployed and as demand changes. This new network interface will be used by service providers to request limited access to a virtualised network segment that can support advanced per-node customisation. For example, a video on demand service provider may request a virtualised network connecting to its customers, and can deploy custom logic to enable content caching right in the access network nodes, it can support the provisioning of multicast trees close to customers, it can also support the proactive triggering of network access handover for its customers. The virtualised network slice provisioned to the service provider can also react to service demand, accumulating more resources in the access network should the service become more popular.

The new network interface, if supported by many network operators, can be used to federate many networks together so that service providers can build a distributed array of virtual network resources and functions that are all orchestrated to support a service with fluctuating demand. In figure 1 we show the potential usage of a new network interface called the Virtual Access Service Interface or VASI.

\section{SCENARIO}

In this section we present a realistic usage of the VASI to realise a new type of service which truly illustrates the benefits of Edge-as-a-Service. A new Mobile Virtual Network Operator called Big Events (Figure 2), targets a niche market of sports enthusiasts. For a one time fee, the MVNO will allow customers to access its value added service called "Match Day" which offers its customers exclusive access network based 


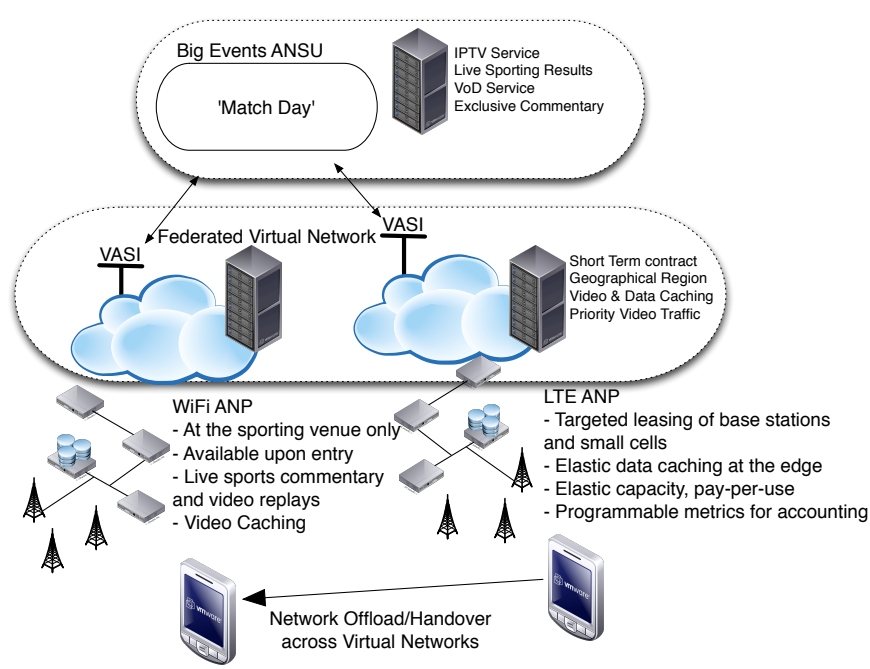

Figure 2. Big Events MVNO seeks to make use of many network providers to deploy a specialised service to its customers. The service requires features such as very low latency, that can only be guaranteed by making use of a network operators provided API.

services. Users can receive a) guaranteed data rates at the venue, b) streamed live action replays to their mobile handsets, c) exclusive access to real-time sports results and commentary, and d) low cost video calls to other customers of the service. To create this service, Big Events must compose and federate resources available from two different network operators. Big Events acts as an Access Network Service User (ANSU) and will access the resources of Access Network Providers (ANP). Specifically, Big Events federates the resources of a national LTE ANP and a venue based WiFi ANP. The same service is available for end users from both providers, and includes automatic and seamless handover for customers at the sporting event venue. The 'Match Day' service is only available on the day of the sporting event, and depending on the event, may have varying demand.

The new virtualised access service interface (VASI) is available from each ANP and is used by Big Events' service management system to programmatically plan and reserve virtualized access network resources to sustain the service for customers. It will involve the creation of a virtual network, where content caching and data metering will be enabled into each access network operators' infrastructure. Through content caching, and distributed service accounting, there is minimal transit IP traffic, and as much traffic as possible is maintained within each of the ANPs networks, thus minimizing network load. Also, the service popularity will impact on how much resources each ANP must make available for use by the service's customers.

Notably, a pay-as-you-use billing model towards the ANSU can be very suitable to Big Events. There is a low overhead involved in setting up the service, and so, it can prove to encourage innovation in the delivery of these types of services in the future. We now analyse the key advantages over current approaches and highlight what is not possible in today's state of the art.

\section{Elastic usage of access network resources}

The scenario highlights how an Access Network Provider (fixed, wireless or a combination) can offer access to its resources in a manner that can be requested on-demand, much like in the Infrastructure-as-a-Service model of cloud computing. However, unlike Cloud Computing, this scenario would enable active sharing of resources at peak times, and the programmatic placement of access network resources, such as content caching and data plane processing. During times of peak usage, the Access Network Provider can dedicate portions of its resources to favour higher paying service users such as Big Events, therefore offering more incentives for such services. The scenario also supports many new innovative business models where time / location sensitive data can aid in deciding how resources should be shared. For example, the 'Match Day' service takes advantage of service provider policies to perform pro-active handovers when in the proximity of federated WiFi access networks.

Delivery of high quality services directly provisioned from a Content Provider or Over The Top Provider

The scenario shows how value added services can be directly offered by a content provider who would typically rely on best-effort, over-the-top network access. This way, the content provider has the capability to directly purchase network access resources, and they can offer differentiated levels of content quality to end-users. In the scenario, video content of action replays is cached into the virtualized access networks, only taking up storage close to customers of the service, but can be used to offer very high definition video with low latency and lower overall network utilization.

\section{Diversification of revenue generation for Access Network Providers}

The scenario illustrates the benefits that may be achieved by Access Network Providers, who have a pre-existing deployment of multiple access network technologies. They should be able to substantially increase overall network revenue by making it much easier to offer secure and managed access to selected parts of their access networks to 1) other network operators, 2) content providers, 3) brands, all playing the role of Access Network Service Users, similarly to such as niche markets as is the case of 'Big Events'. Also network operators can reduce the total cost of ownership of new infrastructure projects by leveraging network sharing that encompasses data offloading.

\section{Challenges beyond State of the Art}

This section highlights the state of the art and future challenges related to realising the concepts of EaaS. We also capture a set of new research directions in each research field analysed. 


\section{A. Challenges for Network Virtualisation}

Network virtualization [3] is currently being viewed as a flexible means for which network operators can cope with the growing demand for high bandwidth, diverse services and network efficiency across their networks. Current approaches to network virtualization in ISP [7] networks are lacking flexibility and also lack the ability to incorporate the virtualization of a wider set of network resources than just connectivity, such as caching, storage, and data plane processing. Layer 2 and Layer 3 (Virtual Private Network) approaches only abstract the actual data forwarding and do not consider flexible resource elasticity, caching of content, dynamic topology optimisation or programmable data plane processing to support load balancing, multicasting, specific application needs or more fine grained access control. More sophisticated techniques including OverQOS and SON (Service Overlay Network) [8], enable an ISP to request services that constitute a virtual network designed for a particular static class of traffic. Recently VXLAN, a technology that tries to overcome the inflexibility of Ethernet based VLAN, is being developed and lead by VMWare. This technology is primarily targeted to support the virtualization of large numbers of overlapping virtual network topologies in data center environments.

Network operators are also investigating the use of network virtualization in an effort to improve the flexibility of their networks. Specifically, the Telco Cloud initiative as followed by the FP7 funded Mobile Cloud Networking project describes the transformation of the network operators' infrastructure towards a cloud-computing infrastructure, where applications can be hosted in the operators network, along with telecommunications specific network management and control systems. These systems would traditionally be run on dedicated and expensive hardware, and thus offers a great potential to reduce the capital and operational expenditure of network operators. That approach is more focused on reducing the cost of capital expenditure for network operators by enabling more functionality to be pushed to commodity hardware in a cloud. However, it is not focused on leveraging an interface to be designed for and used by service providers to dynamically make use of access network resources.

Current approaches to network virtualization do not go far enough and EaaS solutions will require the virtualization of many more comprehensive forms of resources. Fixed and wireless access networks are tailored for vastly different access scenarios. A common resource model abstracting these networks to support effective resource virtualization does not currently exist; however, each technology is defined in the appropriate standards bodies.

New directions in Network Virtualisation:

- New techniques to support the virtualization of access network resources and systems that consider the requirements of dynamic and on-demand resource pooling, virtualization of data plane processing and caching, and virtualization of heterogeneous access network technolo- gies.

- A comprehensive virtual network resource model that can consider the majority of currently deployed access network technologies and coupled systems, including Cable, xDSL, FTTx, Satellite, 3G/4G, Wifi and WiMax.

- A virtual access network resource management system that can mediate requests for resources from many external clients, and can monitor current usage of leased resources for clients.

- Dynamic spectrum allocation based on access network technology demand in geographical regions.

\section{B. Challenges for Network Programmability}

Network programmability is not a new concept and has its origin in active networks research[9], where it describes the application of customised processing by routers and switches on packets that flow through the network. OpenFlow[6] is a networking technology that enables a logically centralized controller to configure per flow forwarding rules into OpenFlow enables devices. The openness of OpenFlow coupled with the ease of adoption has ignited the use of network programmability in solving many difficult networking challenges, specifically seen in data centers and more recently seen in large ISP networks.

OpenFlow is also a possible enabler for network virtualization, where a system called FlowVisor[10] has been shown to enable strong network isolation for multiple virtual networks sharing the same physical resources. However, as argued by Sonkoly et. al [5], FlowVisor does not programmatically manage the distribution of resources across many virtual network slices. They also argue that a more comprehensive solution to the management of the OpenFlow controllers in a more coordinated fashion is required for any large scale deployment of virtual networks.

Openflow is also seen as a very attractive technology for large network operators to support more advanced network traffic management features. Philip et. al [7] explored the use of OpenFlow to realise e-NodeB virtualization in 3G access networks. Their work aimed to increase the ability of network operators to use others network infrastructure to serve end-users, with minimal impact on users. Software defined networking is being explored by network operators to help to streamline their network infrastructures, and to reduce excessive network packet processing. SDN, and network programmability in general, can be used to selectively place key data plane processing elements throughout the access network to help reduce the load of key centralised network components which may be suitable to realise EaaS features in operators networks.

One of the major advantages of being able to control the flow of mobile data in the access network is to improve the utilisation of caching of video data, and to enable more effective usage of elastic resources, as we shall see in the next section.

New directions in Network Programmability: 
- A network programmability solution that can support the separation of control plane from data plane processing in access networks. These are typically centralised in current access networks, and should support lower control overhead which users are roaming on EaaS.

- A framework to support the coordination of distributed network controllers across multiple access networks that can mediate contention for resources and can ensure secure isolation of resources while abiding by business driven objectives.

- Network programmability principles that include data plane processing, such as load balancing, accounting and multicast that do not exist in current SDN standards such as OpenFlow, that will be specifically tailored for use in virtualized access networks.

- More intelligent control over the flow of mobile data across the access network, to help cope with peaks in demand for resources.

\section{Challenges for Elastic and On-demand Service Manage- ment}

Cloud computing has dramatically transformed the way services are delivered over the Internet today. Much like with cloud computing, the EaaS concept enables service providers to make use of the virtualisation to programmatically build a dedicated and elastic virtual network, tailored to reach its customers, with agreed quality of service and access to other virtualized access network resources such as caches, storage and data plane processing. To achieve this, there is a need for dynamic and programmable algorithms, capable of provisioning virtual access resources in face of changing requirements and characteristics of services and users, as well as operating environments. Additionally, there is a need for methods and models that predict changes in resource demands, allowing the system to pro-actively provision the necessary resources, avoiding temporarily suboptimal behaviour.

The virtual network embedding (VNE) [11] problem is concerned with mapping virtual network topologies and resources onto the physical substrate. Its fundamental goal is to maximise the number of admitted virtual network requests. Virtual network embedding can be classified based on several characteristics; such as static versus dynamic and centralised versus distributed. Most algorithms proposed to date are static and centralised. This means that they cannot cope with service, user and network dynamics and require full knowledge of the entire physical network topology. Such algorithms are obviously useless in a large-scale scenario spanning multiple physical networks, where virtual resource provisioning should scale elastically. EaaS would require dynamic and distributed VNE algorithms, capable of handling the federation of virtual networks, spanning multiple network domains.

Predicting service demand based on service usage patterns can help to pro-actively manage virtual resource placements. Time series analysis has previously been applied to this problem, with the assistance of artificial neural networks and reservoir computing. Additionally, some research has focused specifically on popularity prediction of multimedia content. These works typically identify correlations between specific video characteristics and future popularity. Famaey et al.[12] found that analytical models can be used to predict the distribution associated with specific popularity of video contents. These problems are highly relevant to the EaaS problem space, as mobile users are access higher volumes of mobile data and video content.

User mobility prediction is important to future service providers as they can tailor the distribution of resources to pro-actively meet expected user demand, a feature inherent with EaaS. In EaaS, the main interest lies on the behavior of the aggregated users' mobility and its predictability; this way, it would be possible to anticipate the geographical distribution of the connectivity demand over time. EaaS will need to make use of algorithms that not only consider the present virtual network requests, but also those requests more probable to come up in the short-term; consequently, achieving a better long-term distribution of resources.

New directions for Elastic and On-demand Service Management:

- Virtual network embedding algorithms that operate in a dynamic (i.e., elastic) and distributed fashion, capable of handling federated virtual access networks spanning multiple physical infrastructures.

- Virtual network embedding algorithms that can embed large-scale service requests that consider a wider set of high-level virtualized network resources (e.g., content caches or computing resources), into federated virtualized access networks.

- Techniques to maintain and dynamically grow/shrink virtual networks as a function of business objectives, service demand and user mobility patterns. This will be achieved using heuristic based online algorithms that can meet strict timing deadlines but tradeoff against sub-optimal solutions.

- Generic techniques to efficiently re-distribute virtualized network resources based on pro-active prediction of user demand based on examining service usage history.

\section{Challenges for Network Sharing and Agreements}

Regarding the market, the most related business models are those of Mobile Virtual Network Operators (MVNO). Depending on the MVNO value proposition, they may require fine grained access to network resources, such as bandwidth provisioning and QoS, or very coarse access, such as just billing of data usage. In either case, the MVNO is today highly restricted in how it can access network providers' resources. The EaaS proposition is to dramatically improve MVNO access and usage of resources on a wide range of granularity and with the ability to have rich business models that can harness a range of virtualized access network resources. Although the MVNO is not the only customer of EaaS that we envisage, we do consider that they will be among the first adopters of the EaaS solutions. 
MVNOs access to network operators resources has been improved with the emergence of 3GPP[13], where a recent study reveals that the use of policy within 3GPP, and visiting network components enable some more functionality for the MVNO. However, such techniques have not been explored, and will inherit the problems being faced by current network operators. EaaS asserts that a new network interface should be made available, the Virtual Access Service Interface, which will offer a lot more flexibility to MVNOs.

Network Function Virtualization[1] is an emerging concept that is being investigated by telecommunications network operators as a means to significantly reduce their dependency on expensive telecoms network equipment, while taking advantage of cloud computing concepts for service hosting and delivery. The concept should enable a more comprehensive range of features in the future for MVNOs to take advantage of, including the full range of cloud computing features, but in an operators network. However, current initiatives are not focused on defining an interface that is readily accessible to third parties such as the MVNO, and only seek to support current interfaces supported by cloud computing initiatives.

Beside the MVNO, current mobile network operators need to be able to effectively collaborate in an effort to reduce the cost of deploying new infrastructure projects. Network Sharing aims to 1) share construction costs, and 2) potentially share network access. EaaS aims to define a flexible solution for network sharing that can accommodate a wide range of use cases and to promote the wider scale deployment of small cells as a means to share network access and reduce network operators costs. Current network sharing solutions are proprietary but are starting to show major benefits for some network operators, be it in more resilient back-haul links, or dramatic reductions in network costs. Other approaches to enabling a finer grained access to control network resources are often seen from the end user perspective, where mobile applications running on the users handset can issue network directed API to request better services[2]. This is not a scalable approach due primarily to the scale of network state that would need to be maintained.

New directions for Network Sharing and Agreements:

- A Virtual Access Service Interface or VASI that can be used to request the usage of complex "virtualized access network resources" on a large scale.

- Analysis and consistency checking of access network provider management policy sets, deployed to guide the operation and management of virtualized access networks.

- New network sharing frameworks that can operate at varying degrees of granularity.

- Faster and more flexible network agreements to facilitate very short lived virtual networks.

\section{E. Challenges for Access Network Economics}

Current state of the art approaches to access network resource sharing[14], generally lack economic merit, as they do not prioritize users in terms of their utility for the service provider. Recent schemes such as hierarchical bandwidth allocation and utility-based load balancing[14] consider access networks with competing and overlapping access technologies. These approaches are not extensible to the EaaS business models, which need to consider much more comprehensive economic models. Similarly, recent work on auctioning of data paths in heterogeneous wireless networks[15] are not suitable for EaaS, as those approaches are not extensible to consider many more types of virtualized resources to be allocated, over and above just bandwidth.

EaaS also requires the development of new business models. In particular, previous approaches are limited since they do not virtualize the access network resources but rather rely on attempting to establish end-to-end SLAs among multiple networks, which are difficult to automate, negotiate and enforce. This is in sharp contrast with EaaS where the virtualized access network infrastructure greatly simplifies QoS provisioning over the virtualized infrastructure. This creates a novel paradigm of conducting business that empowers novel business models and new network economics issues to be investigated.

New directions for Access Network Economics:

- New economic models for using overlapping heterogeneous fixed and wireless access technologies in competitive and non-competitive systems.

- Innovative access sharing and service provisioning that can provide insight into important regulatory and market issues, such as network transparency and competition; network neutrality; market power and competition issues.

- Market-based resource allocation schemes and policies that take into account both access technology and economic value to ANPs.

- Techniques to mediate many potentially competing requests for virtualized resources, so as to maximise resource utilisation of resources and the benefit of the access network providers.

- Use of network economics and game theory to evaluate the business aspects, and new business models.

\section{CONCLUSION}

Telecommunication network operators are suffering from falling revenues and unsustainable business models, due to significant investments in infrastructure that is difficult to manage cost effectively. This paper presents and analyses the challenges associated to Edge-as-a-Service. This new concept decouples the strict ownership relationship between network operators and their access network infrastructure. Through the development of a new novel network interface, virtual networks and functions can be requested on-demand to support the delivery of more adaptive services. There are significant challenges ahead, with regards to network virtualisation, network programmability, economical network sharing and ondemand elastic network management.

\section{REFERENCES}

[1] P. Bosch, A. Duminuco, F. Pianese, and T. L. Wood, "Telco clouds and Virtual Telco: Consolidation, convergence, and beyond," in 12th 
IFIP/IEEE International Symposium on Integrated Network Management (IM 2011) and Workshops, pp. 982-988, IEEE, May 2011.

[2] L. Baleh, L. Suciu, and J.-M. Bonnin, "Enriched connectivity-as-aservice for dynamic Mobile-Cloud," in 2012 IEEE Consumer Communications and Networking Conference (CCNC), pp. 655-660, IEEE, Jan. 2012.

[3] N. Chowdhury and R. Boutaba, "Network virtualization: state of the art and research challenges," Communications Magazine, IEEE, vol. 47, no. 7, pp. 20-26, 2009.

[4] N. Chowdhury, K. Mosharaf, and R. Boutaba, "A Survey of Network Virtualization,” Computer Networks, vol. 54, pp. 862-876, Apr. 2010.

[5] B. Sonkoly, A. Gulyas, F. Nemeth, J. Czentye, K. Kurucz, B. Novak, and G. Vaszkun, "OpenFlow Virtualization Framework with Advanced Capabilities," in 2012 European Workshop on Software Defined Networking, pp. 18-23, IEEE, Oct. 2012.

[6] N. McKeown, T. Anderson, H. Balakrishnan, G. Parulkar, L. Peterson, J. Rexford, S. Shenker, and J. Turner, "OpenFlow," ACM SIGCOMM Computer Communication Review, vol. 38, p. 69, Mar. 2008.

[7] V. Philip, Y. Gourhant, and D. Zeghlache, "OpenFlow as an Architecture for e-Node B Virtualization," e-Infrastructure and e-Services for Developing Countries, vol. 42, 2012.

[8] A. D. Keromytis, V. Misra, and D. Rubenstein, "SOS: Secure Overlay Services," in Proceedings of the 2002 conference on Applications, technologies, architectures, and protocols for computer communications - SIGCOMM '02, vol. 32, (New York, New York, USA), p. 61, ACM Press, Aug. 2002.

[9] D. Tennenhouse, J. Smith, W. Sincoskie, D. Wetherall, and G. Minden, "A survey of active network research," IEEE Communications Magazine, vol. 35, pp. 80-86, Jan. 1997.

[10] R. Sherwood and G. Gibb, "Flowvisor: A network virtualization layer," Switch Consortium, Tech. Rep, 2009.

[11] A. Fischer, J. F. Botero, M. T. Beck, H. de Meer, and X. Hesselbach, "Virtual Network Embedding: A Survey," IEEE Communications Surveys \& Tutorials, pp. 1-19, 2013.

[12] J. Famaey, F. Iterbeke, T. Wauters, and F. De Turck, "Towards a predictive cache replacement strategy for multimedia content," Journal of Network and Computer Applications, vol. 36, pp. 219-227, Jan. 2013.

[13] R. Copeland and N. Crespi, "Resolving ten MVNO issues with EPS architecture, VoLTE and advanced policy server," in 2011 15th International Conference on Intelligence in Next Generation Networks, pp. 29 34, IEEE, Oct. 2011.

[14] Z. Yu, R. Yanxia, C. Hyeong-Ah, K. Jae-Hoon, S. JungKyo, and C. Hyeong In, "Utility-based load balancing in WLAN/UMTS internetworking systems," in 2008 IEEE Radio and Wireless Symposium, pp. 587-590, IEEE, Jan. 2008.

[15] P. Maillé and B. Tuffin, "Price war in heterogeneous wireless networks," Computer Networks, vol. 54, no. 13, pp. 2281-2292, 2010. 